Vol.: 4 Issue: 2 Date: 31.12.2020 Received: 31.10.2020 Accepted: 27.12.2020 Final Version: 29.12.2020

ISVOS Journal, 2020, 4(2): 86-89 - https://doi.org/10.47897/bilmes.818723

\title{
Mannheim Curves in 3-Dimensional Euclidean Space
}

\author{
Emre Öztürk ${ }^{a, 1}$ \\ a Turkish Court of Accounts, Ankara, 06520, Turkey \\ ORCID ID: 0000-0001-6638-3233
}

\begin{abstract}
In this paper, we consider the Mannheim curve and the slant helix together. We called this curve as a Mannheim slant helix shortly. First we calculate the (first) curvature $\boldsymbol{\kappa}(\boldsymbol{s})$, and the curvature of the tangent indicatrix of the Mannheim curve, in terms of the arc-lenght parameter of the curve. Also, we proved that if the Mannheim curve is also slant helix, i.e. if it is Mannheim slant helix, then the partner curve is general helix. Moreover, we show the striction curve of the ruled surface such that the base curve is Mannheim curve, and the rulings are the normal vector field of the Mannheim curve, is the Mannheim partner curve. Finally, we show the ruled surface such that the base curve is Mannheim curve, and the rulings are the normal vector field of the Mannheim curve is non-developable while the torsion of the Mannheim partner curve $\overline{\boldsymbol{\tau}}(\boldsymbol{s}) \neq \pm \infty$ for all s.
\end{abstract}

Keywords: "Mannheim curve, Slant helix, Ruled surface"

\section{Introduction and Material-Method}

The Mannheim curve is firstly investigated by French mathematician Amédéé Mannheim (1831-1906) in 1878. The Mannheim curve is a space curve such that the principal normal line of the curve coincides with binormal line of the another curve at the corresponding points of the curves. In general, the first curve is called as Mannheim curve and the second curve is called as Mannheim partner curve of the first one. All together they called as a Mannheim pair. It is well known that if $(\alpha, \bar{\alpha})$ is a Mannheim pair then, we can write

$$
\alpha=\bar{\alpha}+\lambda \vec{N}
$$

where $\overrightarrow{\boldsymbol{N}}$ is the normal vector field of $\alpha$ and $\lambda$ is constant.

Also, the curvatures of the Mannheim curve satisfy

$$
\kappa(t)=\lambda\left(\kappa^{2}(t)+\tau^{2}(t)\right)
$$

where $\kappa$ is the curvature, $\tau$ is the torsion of the curve. We note that $\lambda$ is the distance between the corresponding points of the Mannheim pair curve.

Even if the definitions and some basic properties of this curve is given in previous century, most of studies, especially in three dimensional spaces, are given in the recent times. Wang and Liu [1] studied this curve in three dimensional Euclidean and Minkowski space. They stated the first derivative of the torsion of the partner curve depending on the curvatures of the Mannheim curve. Then, Orbay and Kasap [2] gave the torsion of the partner curve in terms of the curvatures of the Mannheim curve by following:

$$
\bar{\tau}(t)=\frac{\kappa(t)}{\lambda \tau(t)}
$$

\footnotetext{
${ }^{1}$ Corresponding author. +90-505-722-3593.
}

E-mail address: emreozturk1471@gmail.com 
Yaylı [3] investigated these curves in three dimensional Minkowski space in detail. Honda and Takahashi [4] stated the curvatures of the partner curve depending on the curvatures of the Mannheim curve as follows:

$$
\bar{\kappa}(s)=\frac{\kappa(s)\left(\kappa(s) \tau^{\prime}(s)-\kappa^{\prime}(s) \tau(s)\right)}{|\lambda \tau(s)|\left(\kappa^{2}(s)+\tau^{2}(s)\right)^{\frac{3}{2}}}, \bar{\tau}(s)=\frac{\kappa^{2}(s)+\tau^{2}(s)}{\tau(s)}
$$

The concept of the slant helix is firstly introduced by Izumiya and Takeuchi [5]. They defined the slant helix as a curve that its principal normal lines make a constant angle with a fixed direction and characterized the slant helices by following:

Proposition 1.1 Let $\gamma$ be a unit speed curve with $\kappa(s) \neq 0$. Then $\gamma$ is a slant helix if and only if

is a constant function.

$$
\sigma(s)=\left(\frac{\kappa^{2}}{\left(\kappa^{2}+\tau^{2}\right)^{\frac{3}{2}}}\left(\frac{\tau}{\kappa}\right)^{\prime}\right)(s)
$$

Let $\mathrm{I}$ be an interval of $\mathbb{R}$ and let $\gamma: \mathrm{I} \rightarrow \mathbb{R}^{3}$ be a regular space curve, that is, $\gamma^{\prime}(\mathrm{t}) \neq 0$ for all $\mathrm{t} \in \mathrm{I}$, where $\gamma^{\prime}(\mathrm{t})=(\mathrm{d} \gamma / \mathrm{dt})(\mathrm{t})$.

We say that $\gamma$ is nondegenerate, or $\gamma$ satisfies the nondegenerate condition if $\gamma^{\prime}(t) \times \gamma^{\prime \prime}(t) \neq 0$ for all $t \in I$.

The surface formed by moving lines along a space curve is called a ruled surface. The moving lines are called the rulings of the surface and the space curve is called the base curve.

Let $\alpha$ be the base curve and $\vec{\gamma}$ be the rulings of the ruled surface. The parametrization of this surface is given by $\psi(s, u)=\alpha(s)+u \vec{\gamma}(s)$.

The striction curve, and the distrubition parameter (drall) of the surface is given by

$$
\beta(s)=\alpha(s)-\frac{\left\langle\alpha^{\prime}(s), \vec{\gamma}^{\prime}(s)\right\rangle}{\left\langle\vec{\gamma}^{\prime}(s), \vec{\gamma}^{\prime}(s)\right\rangle} \vec{\gamma}(s),
$$

and

$$
P_{X}=\frac{\operatorname{det}\left(\alpha^{\prime}(s), \vec{\gamma}(s), \vec{\gamma}^{\prime}(s)\right)}{\left\langle\vec{\gamma}^{\prime}(s), \vec{\gamma}^{\prime}(s)\right\rangle}
$$

respectively.

Throughout this paper, the curves will be considered as an nondegenerate, unless otherwise mentioned.

\section{Results}

In this section, we give our main results about the Mannheim curves and the ruled surfaces.

Theorem 2.1 Let $\alpha$ be an non-degenerate unit speed Mannheim slant helix. The curvature of this curve is given by

$$
\kappa=\frac{1}{\lambda} \operatorname{sech}^{2} \varphi
$$

where $\varphi$ is a linear function of arc-lenght parameter.

Proof. Let $\alpha$ be a Mannheim slant helix. From (1) and (4) we get

$$
\sigma(s)= \pm \frac{\lambda}{2} \frac{\kappa^{\prime}(s)}{\kappa(s) \sqrt{1-\lambda \kappa(s)}}
$$

Since $\sigma(s)$ is constant, $\pm \frac{\lambda}{2} \frac{\kappa^{\prime}(s)}{\kappa(s) \sqrt{1-\lambda \kappa(s)}}$ is also constant. So, we have the differential equation as follows: 


$$
\kappa^{\prime}(s)-c \kappa(s) \sqrt{1-\lambda \kappa(s)}=0
$$

where $c$ is a constant. Solution of (5) gives the intended.

Theorem 2.2 Let $(\alpha, \bar{\alpha})$ be a Mannheim pair curve. If $\alpha$ is a Mannheim slant helix then, the partner curve $\bar{\alpha}$ is general helix.

Proof. If $\alpha$ is a Mannheim slant helix then, $\sigma(s)$ is constant and $\kappa(s)=\lambda\left(\kappa^{2}(s)+\tau^{2}(s)\right)$. We note that $s$ is not the arc-length parameter of $\bar{\alpha}$. Since $\sigma(s)$ is constant, it follows from (3) that

$$
\bar{\kappa}(s)=a \frac{\kappa(s)}{\tau(s)} ; \bar{\tau}(s)=b \frac{\kappa(s)}{\tau(s)}
$$

where $a$ and $b$ are constant. Hence, we have $\frac{\bar{\tau}(s)}{\bar{\kappa}(s)}=\frac{b}{a}$ is constant, and this completes the proof.

Theorem 2.3 Let $\alpha$ be a Mannheim slant helix. The curvature of the tangent indicatrix of $\alpha$ is given by

$$
\kappa_{T}(s)= \pm \cosh \varphi
$$

where $\varphi$ is a linear function of arc-lenght parameter of the curve.

Proof. It is well known that the curvature of the tangent indicatrix of the curve is as follows,

$$
\kappa_{T}(s)=\frac{\sqrt{\kappa^{2}(s)+\tau^{2}(s)}}{\kappa(s)}
$$

where $\kappa(s)$ and $\tau(s)$ are the curvature and the torsion of $\alpha$, respectively. By Theorem 2.1, we get the intended.

Proposition 2.4 Let $\gamma$ be an unit speed Mannheim curve and $\vec{N}$ be the normal vector field of $\gamma$ in $\mathbb{R}^{3}$. The striction line of the ruled surface

$$
\psi(s, u)=\gamma(s)+u \vec{N}(s)
$$

is the Mannheim partner curve of $\gamma$.

Proof. The striction line of the ruled surface $\psi$ is given by

$$
\beta(s)=\gamma(s)-\frac{\left\langle\gamma^{\prime}(s), \overrightarrow{\boldsymbol{N}^{\prime}}(s)\right\rangle}{\left\langle\overrightarrow{\boldsymbol{N}^{\prime}}(s), \overrightarrow{\boldsymbol{N}^{\prime}}(s)\right\rangle} \overrightarrow{\boldsymbol{N}}(s) .
$$

Since $\kappa(s)=\lambda\left(\kappa^{2}(s)+\tau^{2}(s)\right)$, we get $\beta(s)=\gamma(s)+\lambda \overrightarrow{\boldsymbol{N}}(\boldsymbol{s})$ which completes the proof.

If the distrubition parameter of a ruled surface is vanishing, that surface is said to be developable, otherwise it is said to be non-developable or skew. In the following theorem, we show that the ruled surface is non-developable, under the certain condition.

Theorem 2.5 Let $\gamma: I \rightarrow \mathbb{R}^{3}$ be an unit speed Mannheim curve and $\overrightarrow{\boldsymbol{N}}$ be the normal vector field of $\gamma$. Suppose that the torsion of the Mannheim partner curve $\bar{\tau}(s) \neq \pm \infty$ for all $s$. Then, the ruled surface of

$$
\psi(s, u)=\gamma(s)+u \vec{N}(s)
$$

is non-developable.

Proof. By straightforward calculations, we get the distribution parameter of $\psi$ as

$$
P_{X}=\frac{\operatorname{det}\left(\gamma^{\prime}(s), \overrightarrow{\boldsymbol{N}}(s), \overrightarrow{\boldsymbol{N}^{\prime}}(s)\right)}{\left\langle\overrightarrow{\boldsymbol{N}^{\prime}}(s), \overrightarrow{\boldsymbol{N}^{\prime}}(s)\right\rangle}=\frac{\tau(s)}{\kappa^{2}(s)+\tau^{2}(s)}
$$


It follows from (1) and (2) that

$$
\bar{\tau}(s)=\frac{\kappa(s)}{\lambda \tau(s)}=\frac{\kappa^{2}(s)+\tau^{2}(s)}{\tau(s)}
$$

From (6) and (7) we get $P_{X}=\frac{1}{\bar{\tau}(s)}$. Since $\bar{\tau}(s) \neq \pm \infty$ for all s, $P_{X} \neq 0$ and $\psi$ is non-developable.

\section{Conclusion}

In this study, we considered the some invariant properties of the Mannheim slant helix, for instance the curvature of the curve is calculated by its arc-lenght parameter. Also, we gave the curvature of the tangent indicatrix of the Mannheim curve by arclenght parameter similarly. Moreover, we showed that the striction curve of the the ruled surfaces such that the base curve is Mannheim curve and the rulings are the normal vector field of the Mannheim curve is the Mannheim partner curve. Finally, we proved that the ruled surface is non-developable if the torsion of the Mannheim partner curve $\bar{\tau}(s) \neq \pm \infty$ for all s.

For further studies, these curves will be considered and will be examined by different frames, and the ruled surfaces will be investigated by taking its base curve as a Mannheim curve in another space. As we did in this study, some curves will be considered together.

\section{Acknowledgment}

The author would like to thank the referees for carefully reading which helped to improve the manuscript.

\section{References}

[1] F. Wang and H. Liu "Mannheim partner curves in 3-space," Proceedings of the eleventh international workshop on differential geometry'11, pp. 25-31, 2007.

[2] K. Orbay and E. Kasap, “On Mannheim partner curves in $\mathbb{E}^{3}$," International Journal of Physical Sciences, vol. 4, no. 5, pp. 261-264, 2009.

[3] Y. Yayl1 "Mannheim slant helix in Lorentz-Minkowski space," International meeting on Lorentzian Geometry,'IX, p. 23,2018 .

[4] S. Honda and M. Takahashi, "Bertrand and Mannheim curves of framed curves in the 3-dimensional Euclidean space," Turkish Journal of Mathematics, vol. 44, no. 3, pp. 883-899, 2020.

[5] S. Izumiya and N. Takeuchi "New special curves and developable surfaces," Turkish Journal of Mathematics, vol. 28, no. 2, pp. 153-163, 2004. 